\title{
Unusual Intestinal Involvement by Paracoccidioidomycosis Diagnosed After Oral Manifestation
}

\author{
Rodrigo Neves-Silva • Patrícia Maria Fernandes • Alan Roger Santos-Silva • \\ Pablo Agustin Vargas $\cdot$ Ricardo de Souza Cavalcante $\cdot$ Marcio Ajudarte Lopes
}

Received: 3 October 2017/ Accepted: 8 February 2018/Published online: 16 February 2018

(C) Springer Science+Business Media B.V., part of Springer Nature 2018

\begin{abstract}
Paracoccidioidomycosis is a common deep fungus infection in South America, particularly in Brazil. It is acquired through inhalation and primary involvement of lungs. Subsequently, dissemination may occur and oral mucosa is frequently affected and actually, in most of the cases the diagnosis is established because of the oral lesions. Thus, the role of the dentist is fundamental to correct diagnosis. However, the involvement of intestine is rarely reported. The current case describes a 36-year-old man who presented abdominal pain and intestinal constipation, being suspected and then confirmed as paracoccidioidomycosis after already be diagnosed with this disease by a dentist through oral manifestations.
\end{abstract}

R. Neves-Silva · P. M. Fernandes · A. R. Santos-Silva · P. A. Vargas - M. A. Lopes

Oral Diagnosis Department, Semiology and Oral Pathology Areas, Piracicaba Dental School, University of Campinas (UNICAMP), Av. Limeira, 901, Piracicaba, SP CEP 13.414-903, Brazil

R. de Souza Cavalcante

Department of Tropical Diseases, School of Medicine, São Paulo State University (UNESP), Av. Prof. Montenegro, s/n, Botucatu, SP CEP: 18618-687, Brazil

M. A. Lopes ( $\square)$

Faculdade de Odontologia de Piracicaba, Departamento de Diagnóstico Oral - Semiologia, UNICAMP, Av. Limeira 901, Piracicaba, SP CEP 13.414-903, Brazil e-mail: malopes@fop.unicamp.br
Keywords Paracoccidioidomycosis - Oral cavity · Intestine $\cdot$ Diagnosis

\section{Introduction}

Paracoccidioidomycosis (PCM) is an endemically fungal infection in Latin America, with higher prevalence in Brazil. It is caused by dimorphic fungi of the genus Paracoccidioides, and the human infection was first described in 1908 by Adolph Lutz [1-8]. Geographic areas of highest incidence are typically humid, high-rainfall areas with acidic soil conditions. The organisms are present in the soil and enter the body mainly through the aerodigestive tract, especially through the lungs $[5,9,10]$. At room temperature, it has a mycelium form that transforms into yeast-like form, the pathogenic form, at $35-37{ }^{\circ} \mathrm{C}$ [8].

The infection induces a host immune response that leads to a large spectrum of clinical presentations, being more common to affect the lungs, mucous membranes of the upper aerodigestive tracts and skin in its chronic form [11, 12]. The involvement of the gastrointestinal tract is rarer, representing 10 to $30 \%$ of the cases [4].

When the PCM affects the intestine, all segments can be involved, but predominantly affects the jejunum, terminal ileum, appendix, and proximal portion of the large intestine. Mesenteric ganglion infarction with lymphatic stasis and lymph reflux 
toward the intestinal lumen was observed in the small and large intestine, leading to an exudative enteropathy $[5,13,14]$. The hematogenic spread is the main form which PCM reaches any organ, include the intestine, but ingestion may be considered [15].

In PCM, polymorphic manifestations are frequently observed, which impairs the establishment of a diagnosis. Thus, PCM is not always suspected and sometimes patients can be misdiagnosed, mainly in cases of females and younger individuals $[1,4,5,16-19]$.

Up to $50 \%$ of the cases present manifestation of PCM in oral mucosa and these oral lesions are very suggestive of the disease. Regarding the local of oral involvement, upper gingiva, soft palate, tongue, and lips are the most common sites $[1,8,19]$. The classical clinical presentation is a superficial ulcer with granular appearance and hemorrhagic points [8]. The clinical manifestations of PCM have been classified as acutesubacute form and chronic form. In the chronic form, most of the patients are men, older than 30 years, with report a prolonged clinical course $[4,8,17,20]$.

The cure rate is high when adequate treatment is readily administered, and mycological cure can be achieved with sulfonamides, azole compounds, or amphotericin [7, 21].

PCM is relevant to dentistry because lesions may involve especially the head and neck, typically the facial skin, nasal mucosa, and oral mucosa [19]. This case report describes a patient who presented oral manifestations of PCM and, afterward evolved with abdominal symptoms leading to the suspicion of an unusual presentation of PCM in the intestine.

\section{Case Report}

A 36-year-old man was referred to our clinic with a chief complaint of oral ulceration with 3 months of evolution and weight loss of $20 \mathrm{~kg}$ along this time. In his medical history, the patient reported treatment with a physician 1 year ago because of intestinal infection and anemia which were treated with metronidazole and ferrous sulfate.

On extra-oral examination, it was observed on palpation, bilateral submandibular, cervical and submental lymph nodes enlargement (Fig. 1a). In addition, there was an ulcerated lesion on the mental skin with involvement of inferior lip (Fig. 1b). Intraorally, multiple ulcers on inferior labial mucosa, left buccal mucosa, floor of mouth, inferior alveolar ridge and soft palate were present (Fig. 1c, d). The patient reported that the oral ulcers were continuously increasing causing dysphagia.

PCM was the main clinical hypothesis of diagnosis based on the clinical features of the lesions. Although the patient has reported that he does not work with agriculture or gardening, he has confirmed to live in an endemic area in Sao Paulo, Brazil. Incisional biopsy was performed in labial mucosa and the histopathological analysis showed pseudoepitheliomatous hyperplasia, chronic inflammation, giant cells and round structures compatible with fungus, visible in hematoxylin and eosin stain (Fig. 2a, b). Histological section stained by Groccott method showed a structure similar to Mickey Mouse ears corresponding to yeast cells with some buds (Fig. 2c). All these findings led to the diagnosis of PCM.

The patient was referred to an infectologist, who prescribed itraconazole $100 \mathrm{mg}$, twice a day. At this time, the lungs were free of lesions on the chest radiography (Fig. 3a) and there were no respiratory symptoms. The search for specific anti-P. brasiliensis, as measured by double agar gel immunodiffusion reaction, showed positivity with titers of $1 / 64$.

Two weeks after starting treatment, the patient developed abdominal pain in colic associated with diarrhea. The abdomen was tense on palpation with painful abrupt decompression in the right iliac fossa. Hydro-aerial noises were increased, and there were no visceromegalies. Abdominal tomography revealed concentric thickening of the left lateral wall of the rectum, suggesting a vegetative lesion, evidenced by contrast injection, showing signs of subocclusion (Fig. 3b). Then, the patient underwent a colonoscopy that showed the presence of an extensive lesion, located at $10 \mathrm{~cm}$ of the anus with papillary, sessile and friable appearance, affecting around $70 \%$ of the intestinal wall although it did not obstruct the canal light. An incisional biopsy was performed, and the histopathological examination of this lesion revealed colonic mucosa with intense chronic granulomatous inflammatory process, with neutrophils and fungal structures of Paracoccidioides spp. Once the patient already had the PCM diagnosis through the histopathological examination of the oral lesions, the antifungal therapy previously established was 

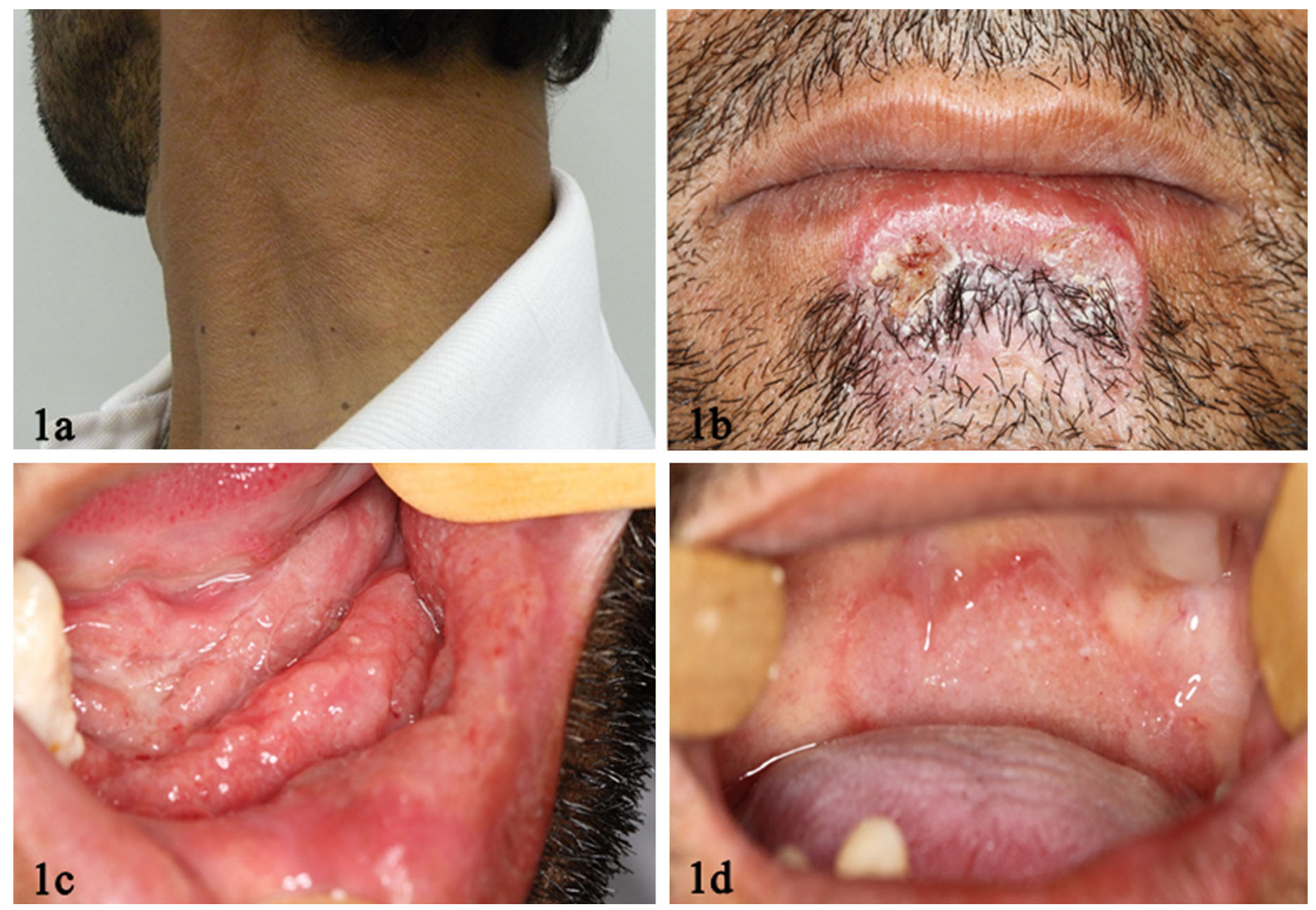

Fig. 1 Clinical presentation. a Palpable lymph nodes in the neck. b Extra-oral ulcerated lesion in the perioral skin. c Extensive 'mulberry-like appearance' lesion on inferior alveolar ridge and floor of mouth. d Ulceration on soft palate with granular surface

maintained and abdominal symptoms were solved with clinical management.

In the third month of antifungal therapy, the patient evolved presenting vomit and return of abdominal pain. The radiography revealed dilatation of intestinal loops, with absence of gauzes in the terminal portion of the intestine (Fig. 3c). The patient was diagnosed with obstructive acute abdomen and underwent exploratory laparotomy which showed thickening of the terminal ileum, adherence of the cecum with duodenum and ileus and absence of the cecal appendix. Right hemicolectomy was performed, whose histopathological examination showed intestinal fibrous adherence, probably caused by the treatment, intestinal perforation, fibrin-leukocyte peritonitis in organization and absence of PCM. About $45 \mathrm{~cm}$ of the large intestine was removed, the patient recovered well and the bowel habit returned to normal.

Some months later, the patient returned for evaluation and all oral and skin lesions have healed. Though it was possible to detect areas of scar fibrosis in the oral mucosa, they were not impeding the normal feeding.

After 18 months from the start of treatment, the patient presented serological cure based on negative result at double agar gel immunodiffusion for PCM and absence of signs and symptoms concluding, therefore, the therapy.

The patient is in follow-up for 3 years, and no signs of recurrence, in any organ, were observed (Fig. 4a, b). The PCM of this patient was classified as severe due to high weight loss, high antibody titers measured by double agar gel immunodiffusion and severe intestinal involvement.

\section{Discussion}

PCM is the most prevalent systemic mycosis in Latin America [2, 21]. Most of the patients are from Colombia, Venezuela, Uruguay, Argentina, 


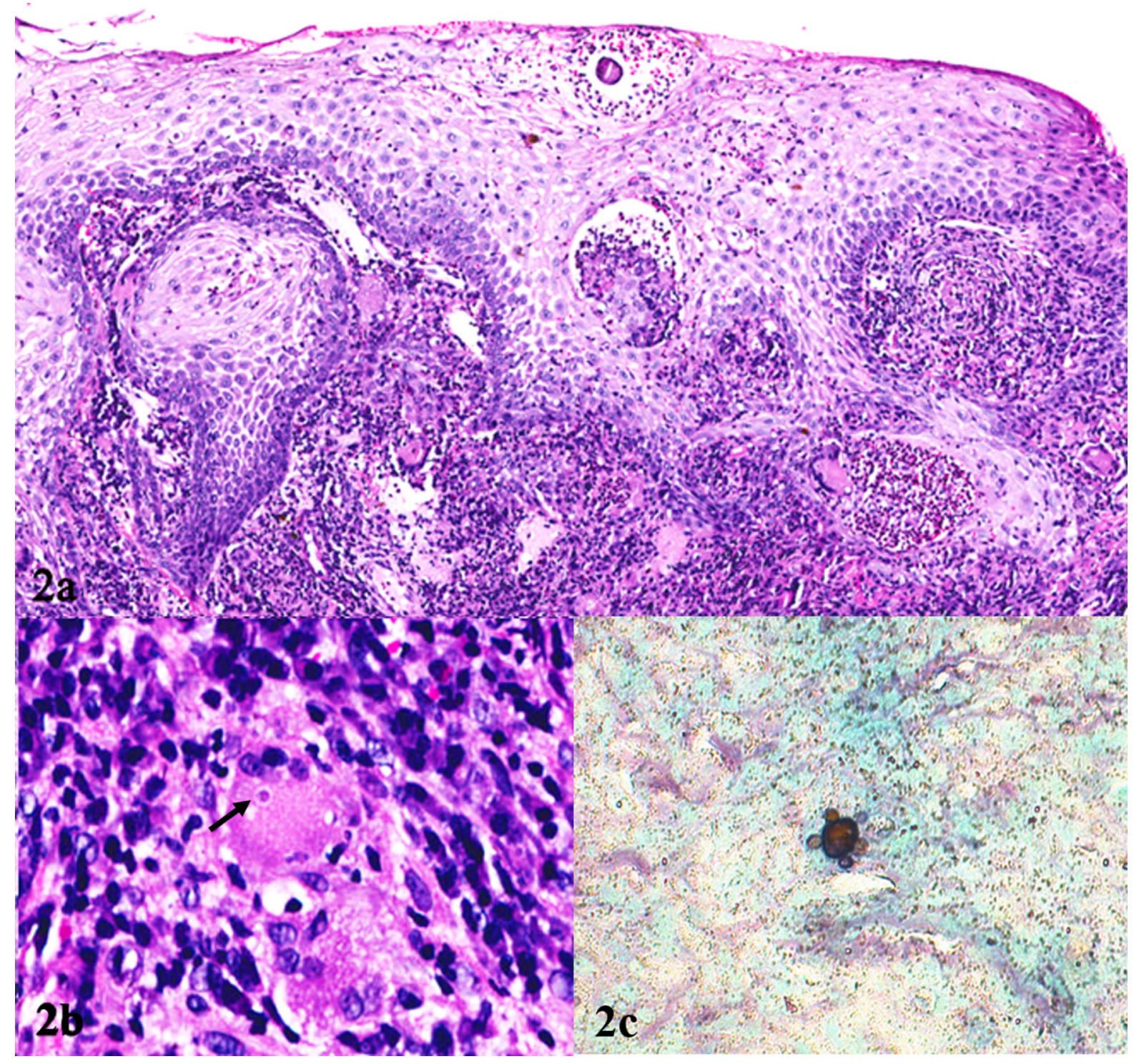

Fig. 2 Histopathological presentation. a Pseudoepitheliomatous hyperplasia, multinucleated giant cells and an intense inflammatory infiltration (hematoxylin and eosin, $\times 50$ ). b High magnification of a giant cell with Paracoccidioides Brasiliensis

Guatemala, and Brazil, where it can be endemic in certain areas, especially in Sao Paulo, Rio de Janeiro, and Minas Gerais States [17, 19]. PCM is seen rarely outside South America, and these cases are generally in patients who have visited the continent. Most affected individuals are adult male with ages ranging from 30 to 59 years, which corresponds to $90 \%$ of the cases. The ratio men/women is $15: 1[5,17,22]$. The disease is rare in children and teenagers, and in these (hematoxylin and eosin, $\times$ 400). c Methenamine silver staining, showing the appearance of fungus, similar to helm, with budding yeasts. (magnification $\times 40$ )

age groups equally affects girls and boys [16]. The patient related here is an inhabitant of an endemic area in Sao Paulo, Brazil, and was 36-year-old at the diagnosis, similar to most of the affected patients.

The highest risk factors for PCM are activities related to the handling of soil contaminated with fungus, such as agricultural activities, gardening, and transport of vegetable products $[3,5,8]$. The professional activity can influence the prevalence of PCM. A 

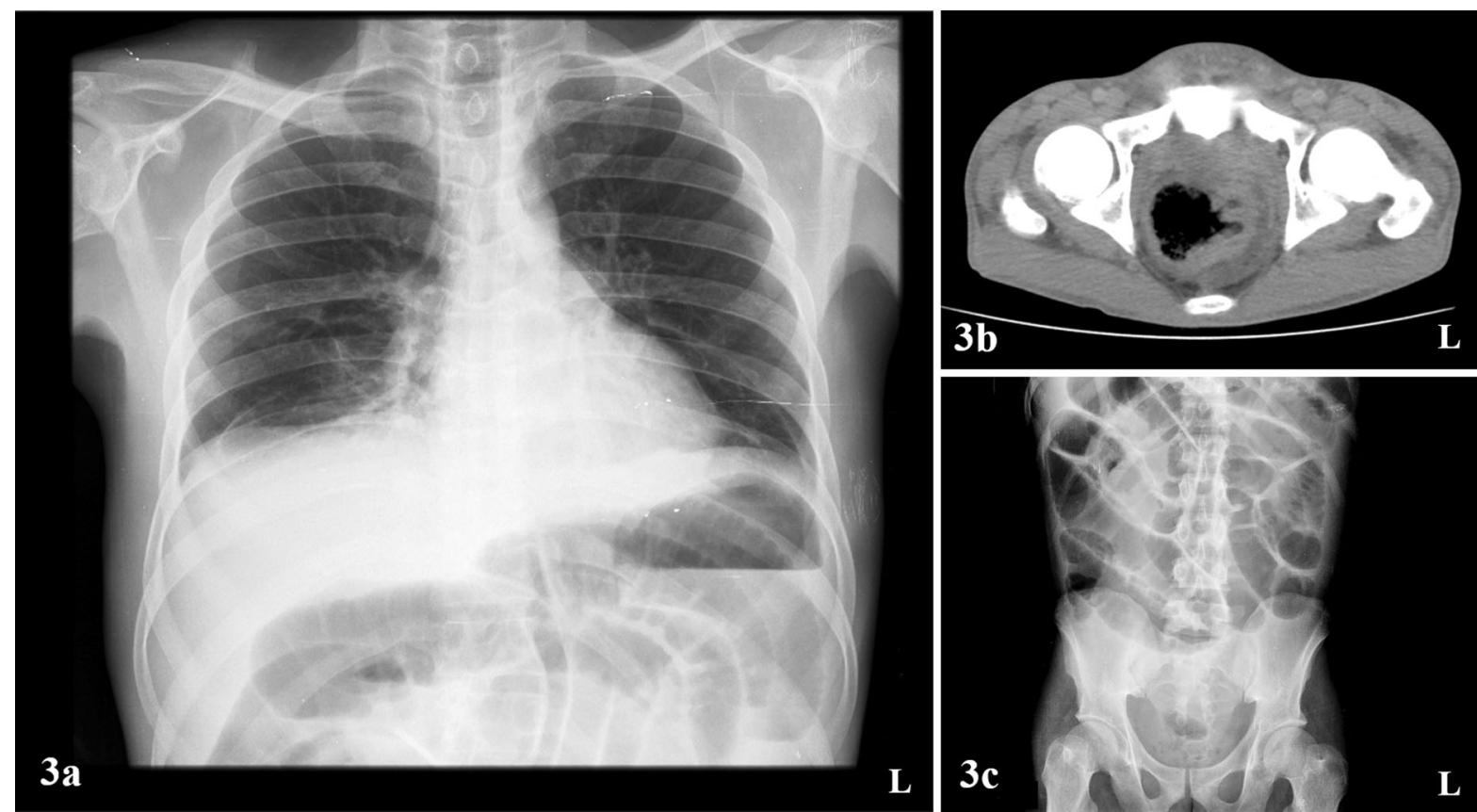

Fig. 3 Imaging examination. a Chest radiography showing absence of pulmonary lesions suggestive of PCM. b Abdominal computed tomography revealed concentric thickening of the left

lateral wall of the rectum, highlighting a vegetative appearance lesion. c Abdominal radiography showing dilatation of intestinal loops, reinforcing the acute abdomen diagnosis
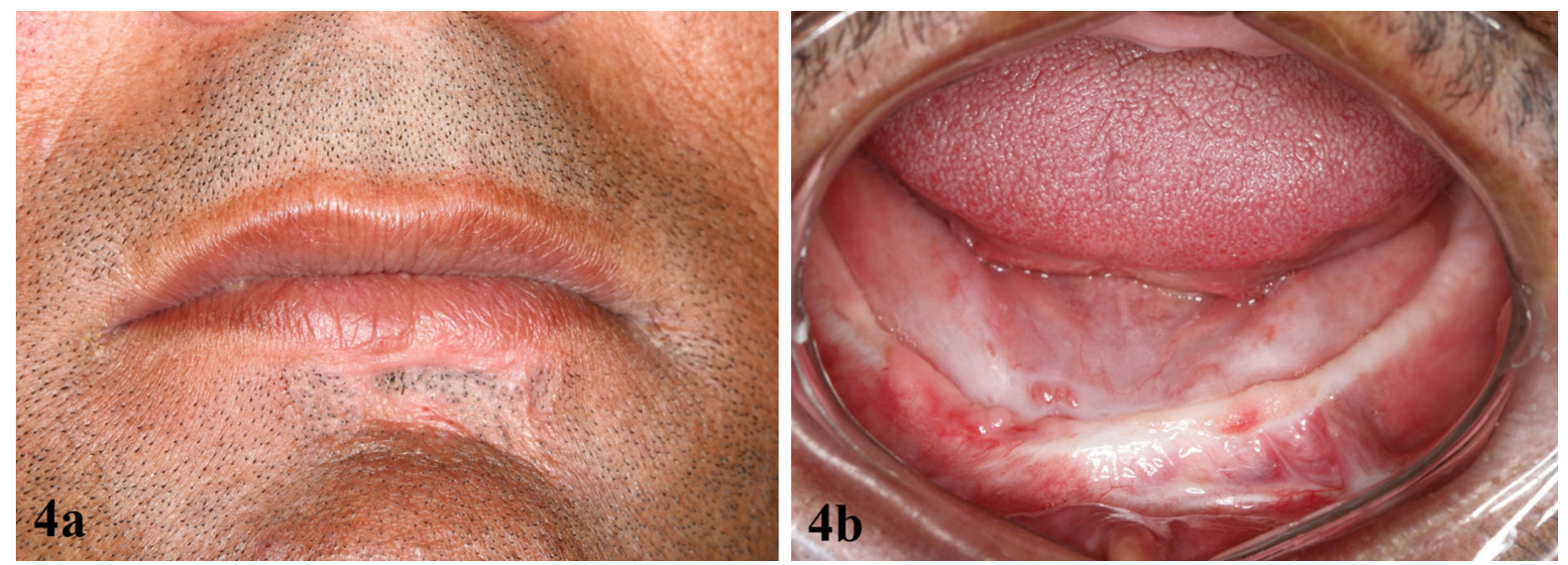

Fig. 4 Clinical presentation in last follow-up. a Absence of perioral lesions and $\mathbf{b}$ intraoral lesion

previous study showed that $39 \%$ of patients diagnosed with PCM have worked with activities related to agriculture $[1,17]$. A previous case series showed that the number of patients was in low-income occupations involving outdoor work such as rural worker (53\%), and the other involved patients were immigrants or excursionists (47\%) [9]. The diagnostic suspicion of PCM of the patient reported here came up based on the clinical and epidemiological features, although he did not work with any of these activities related above.

PCM rarely involves the intestines [5]. Duodenal PCM has been described in approximately $3 \%$ of the autopsied patients with PCM. The intestinal involvement in PCM can affect the mucosa, submucosa, and lymphatic abdominal system and is more frequently seen in the acute/subacute form [5]. Authors has been suggested that the fungi reach the gastrointestinal 
mucosa by the hematogenous route from the lungs and mediastinal lymph nodes, either from the primary infection in subacute form patients or from reactivated lesions in chronic form patients [13-23].

Clinical manifestations of intestinal PCM include epigastric pain and diarrhea for several weeks to several months. Nausea, vomiting, and abdominal distension are symptoms reported by patients with intestinal involvement [5, 13, 17]. Some of these symptoms were present in current patient such as abdominal pain and intestinal constipation. The patient also presented enlargement of cervical lymph nodes and oral lesions.

The diagnosis of PCM is suspected based on the clinical features and confirmed by the identification of the fungus, although this patient had no visible lung lesions on chest radiography. Several methods can be used such as culture and direct mycologic. However, the histopathological analysis is the most common and shows intense inflammatory infiltrate and granulomas rich in epithelioid and giant cells with a double-wall parasite with simple or multiple gemmulations. Pseudoepitheliomatous hyperplasia is a frequent finding $[8,18]$.

In contrast to intestinal manifestations, oral lesions of PCM are much more common and incisional biopsy and exfoliate cytology are efficient for an early diagnosis of this disease [1, 19]. Grocott-Gomori, methenamine silver nitrate or periodic acid-Schiff stains may be superior to hematoxylin and eosin stain in detecting the fungi $[9,19]$. Serology helps to confirm the diagnosis and to monitor treatment. The diagnosis of PCM in the current patient was established, firstly, through oral biopsy and histopathological analysis. When the patient came to present intestinal symptoms, the previously established diagnosis helped in the correct and fast management of the patient, considering that all the signs and symptoms were part of the same disease.

The treatment of PCM is based on systemic antifungal therapy and there are several drugs that are able to treat the disease. The most commonly used drugs are itraconazole, cotrimoxazole, and amphotericin B. The period of the therapy used to be long, with a minimum of 9 and maximum of 18 months, being 1 year of treatment the average duration. This period must be enough to eliminate signs and symptoms, normalize image exams, control weight loss and have negative results in serological tests because those agents cited above can arrest disease progress, but relapses can occur unless continuous therapy is used $[11,12,18,21]$.

Typically, there is marked clinical improvement after 2 months of treatment with healing of mucous lesions [3]. The patient presented here was treated with itraconazole, $200 \mathrm{mg}$, daily, for 18 months. Although he has undergone to hemicolectomy, he had a good outcome in all involved sites, presenting healing of all lesions in mouth, skin, and intestine, aside to present a negative result in immunodiffusion test after completing therapy. In this case, there was no involvement of the lung observed in the chest radiography.

This intestinal involvement, with lack of lung manifestation, could be explained by the fact that the primary infection, which occurs in the lungs, is assaulted by the immune response, resulting in a silent process and control of the fungus leading to absence of respiratory symptoms or clinical signs able to be detected in the chest radiography. The literature points that, even after the immune response, some yeast cells that are viable, remain in the affected tissue and they are able to spread themselves reaching other organs using the lymphatic system or hematogenic route, affecting the gastrointestinal tract, in some cases. All this process result in a chronic form of the disease, which originates from reactivated foci far away from lungs, possibly due to an initial dissemination of the yeast cells [4].

The clinical prognosis in PCM depends on the initial severity of the disease, the presence of comorbidities, as well as the therapeutic schedule used [3]. In pulmonary parenchyma, even after treatment, $P$. brasiliensis induces chronic damage that leads to the development of lung fibrosis, which is most likely because of persistent antigenic stimulus that elicits a continuous inflammatory response [7, 24]. Similar situation occurred with the patient presented here. During the therapy, he developed an acute abdomen and the colonoscopy showed areas of fibrosis, probably due to the treatment. After the hemicolectomy, the patient recovered well and remains without evidence of recurrence. About the prognosis of intestinal PCM specifically, it is clear that the early diagnosis is essential to a good outcome. In a report recently published, four patients were diagnosed with intestinal PCM, being that 1 of them have abandoned the treatment and did not return to revaluation. 
Another one was positive to HIV, which may have precipitated the manifestation of PCM in an unusual place but, just as the two others patients reported, with the treatment performed properly, obtained a good response [25]. The patient reported here was negative to HIV.

In conclusion, patients with oral PCM should be carefully evaluated in order exclude the involvement of other sites. Although rare, intestinal PCM manifestation may occur and the proper diagnosis is essential for successful treatment and disease control.

\section{Compliance with Ethical Standards}

Conflict of interest The authors declare that they have no conflict of interest.

\section{References}

1. Azenha MR, Caliento R, Brentegani LG, de Lacerda SA. A retrospective study of oral manifestations in patients with paracoccidioidomycosis. Braz Dent J. 2012;23(6):753-7.

2. Amorim Pellicioli AC, Neves-Silva R, Santos-Silva AR, Vargas PA, Lopes MA. Synchronous oral paracoccidioidomycosis and pulmonary tuberculosis in an immunocompetent patient. Mycopathologia. 2015;179(5-6):459-64.

3. Marques SA. Fungal infections of the mucous membrane. Dermatol Ther. 2010;23(3):243-50.

4. Benard G, Costa AN, Leopercio AP, Vicentini AP, Kono A, Shikanai-Yasuda MA. Chronic paracoccidioidomycosis of the intestine as single organ involvement points to an alternative pathogenesis of the mycosis. Mycopathologia. 2013;176(5-6):353-7.

5. Berni AD, Elias Junior E, Arao Filho A, Paniago AM. Protein-losing enteropathy in paracoccidioidomycosis identified by scintigraphy: report of three cases. Braz J Infect Dis. 2010;14(5):540-3.

6. Brunaldi MO, Rezende RE, Zucoloto S, Garcia SB, Modena JL, Machado AA. Co-infection with paracoccidioidomycosis and human immunodeficiency virus: report of a case with esophageal involvement. Am J Trop Med Hyg. 2010;82(6):1099-101.

7. Costa AN, Benard G, Albuquerque AL, et al. The lung in paracoccidioidomycosis: new insights into old problems. Clinics. 2013;68(4):441-8.

8. de Oliveira Gondak R, Mariano FV, dos Santos Silva AR, Vargas PA, Lopes MA. Single oral paracoccidioidomycosis mimicking other lesions: report of eight cases. Mycopathologia. 2012;173(1):47-52.

9. Bicalho RN, Santo MF, de Aguiar MC, Santos VR. Oral paracoccidioidomycosis: a retrospective study of 62 Brazilian patients. Oral Dis. 2001;7(1):56-60.
10. Londero AT, Ramos CD, Lopes JO. Progressive pulmonary paracoccidioidomycosis a study of 34 cases observed in Rio Grande do Sul (Brazil). Mycopathologia. 1978;63(1):53-6.

11. Azevedo RS, Gouvea AF, Lopes MA, Correa MB, Jorge J. Synchronous oral paracoccidioidomycosis and oral squamous cell carcinomas with submandibular enlargement. Med Mycol. 2011;49(1):84-9.

12. Almeida OP, Jorge Junior J, Scully C. Paracoccidioidomycosis of the mouth: an emerging deep mycosis. Crit Rev Oral Biol Med. 2003;14(4):268-74.

13. Fonseca LC, Mignone C. Paracoccidioidomycosis of the small intestine. Radiologic and anatomo clinical aspects of 125 cases. Rev Hosp Clin Fac Med Sao Paulo. 1976;31(3):199-207.

14. Gava P, de Melo AS, Marchiori E, Costa MH, Pereira E, Rangel RD. Intestinal and appendiceal paracoccidioidomycosis. Radiol Bras. 2015;48(2):1267.

15. Bravo EA, Zegarra AJ, Piscoya A, Pinto JL, de Los Rios RE, Prochazka RA, Huerta-Mercado JL, Mayo NL, Tagle M. Chronic diarrhea and pancolitis caused by paracoccidioidomycosis: a case report. Case Rep Med. 2010;2010:140505.

16. Penna FJ. Blastomycosis of the colon resembling clinically ulcerative colitis. Gut. 1979;20(10):896-9.

17. Goldani LZ. Gastrointestinal paracoccidioidomycosis: an overview. J Clin Gastroenterol. 2011;45(2):87-91.

18. Ramos ESM, Saraiva Ldo E. Paracoccidioidomycosis. Dermatol Clin. 2008;26(2):257-69.

19. de Almeida OP, Jorge J, Scully C, Bozzo L. Oral manifestations of paracoccidioidomycosis (South American blastomycosis). Oral Surg Oral Med Oral Pathol. 1991;72(4):430-5.

20. Franco M, Montenegro MR, Mendes RP, Marques SA, Dillon NL, Mota NG. Paracoccidioidomycosis: a recently proposed classification of its clinical forms. Rev Soc Bras Med Trop. 1987;20(2):129-32.

21. Shikanai-Yasuda MA, Mendes RP, Colombo AL, QueirozTelles F, Kono ASG, Paniago AM, et al. Brazilian guidelines for the clinical management of paracoccidioidomycosis. Rev Soc Bras Med Trop. 2017. https://doi.org/10. 1590/0037-8682-0230-2017.

22. de Castro LF, Ferreira MC, da Silva RM, Blotta MH, Longhi LN, Mamoni RL. Characterization of the immune response in human paracoccidioidomycosis. $\mathrm{J}$ Infect. 2013;67(5):470-85.

23. Paniago AM, Aguiar JI, Aguiar ES, et al. Paracoccidioidomycosis: a clinical and epidemiological study of 422 cases observed in Mato Grosso do Sul. Rev Soc Bras Med Trop. 2003;36(4):455-9.

24. Restrepo S, Tobon A, Trujillo J, Restrepo A. Development of pulmonary fibrosis in mice during infection with Paracoccidioides brasiliensis conidia. J Med Vet Mycol. 1992;30(3):173-84.

25. Alcántara Figueroa C, Verástegui R, Aphang Lam M, Cáceres Alpaca D. Colon Paracoccidioidomycosis in a hospital from Lima-Peru: report of 4 cases. Ver Gastroenterol Peru. 2017;37(1):77-81 (Spanish). 